\title{
Supramalleolar osteotomy with medial distraction arthroplasty for ankle osteoarthritis with talar tilt
}

\author{
Hong-Mou Zhao, Xiao-Dong Wen, Yan Zhang, Jing-Qi Liang, Pei-Long Liu, Yi Li, Jun Lu and Xiao-Jun Liang*
}

\begin{abstract}
Background: An increased preoperative talar tilt (TT) angle was reported to be positively correlated with treatment failure after supramalleolar osteotomy (SMOT) for varus ankle osteoarthritis. Distraction arthroplasty was reported to have the ability to correct increased $\Pi$ angles. The purpose of the current study was to compare the outcomes between SMOT with and without medial distraction arthroplasty (MDA) in the treatment of varus ankle osteoarthritis with increased $\Pi$ angles.
\end{abstract}

Methods: We retrospectively reviewed the functional outcomes and radiological findings of 34 patients who underwent SMOT with or without MDA for varus ankle osteoarthritis with increased $T$ angles. The American Orthopaedic Foot and Ankle Society (AOFAS) ankle-hindfoot score and Ankle Osteoarthritis Scale (AOS) scores were used for functional evaluation. The tibial anterior surface (TAS) angle, talar tilt (TT) angle, tibial medial malleolar (TMM) angle, talocrural (TC) angle, tibial lateral surface (TLS) angle, and hindfoot alignment (HFA) angle were evaluated preoperatively and at the time of the last follow-up.

Results: In the SMOT group, the AOFAS score and AOS pain and function scores were significantly improved ( $P<0.01$ for each) at a mean follow-up of 61 months. The TAS, TT, TC, TLS, and HFA angles were all significantly improved $(P<0.01$ for each). Similarly, in the SMOT with MDA group, the AOFAS score, AOS pain and function scores, and the TAS, TT, TC, TLS, and HFA angles were all significantly improved postoperatively ( $P<0.01$ for each). When comparing the two groups, the postoperative $\Pi$ angle was significantly smaller in the SMOT with MDA group $(P=0.023)$ than in the SMOT group. In addition, the failure rate of $\Pi$ angle correction was significantly higher in the SMOT group $(P=0.016)$ than in the SMOT with MDA group.

Conclusion: SMOT is a promising procedure for functional improvement and malalignment correction for varus ankle osteoarthritis, even in patients with increased talar tilt. If SMOT is combined with MDA, there can be an improvement in the correction of the increased talar tilt.

Level of evidence: Level III, a retrospective comparative study

\section{Background}

Ankle osteoarthritis is a progressive disease characterized by the degeneration of articular cartilage and often develops asymmetrically with a concomitant varus deformity $[1,2]$. The uneven pressure on the articular surface has a close relationship with the degeneration of cartilage, which may induce osteoarthritic changes and the progression and development of the disease [3]. Supramalleolar

\footnotetext{
* Correspondence: honghuizhm@163.com

Foot and Ankle Surgery Department, Honghui Hospital of Xi'an Jiaotong University, Xi'an 710054, China
}

osteotomy (SMOT) is based on the theory of joint pressure redistribution during weight-bearing actions and aims to delay the progression and development of osteoarthritis $[4,5]$. Clinical and biomechanical studies reported that SMOT could realign the weight-bearing line, restore the congruence of the tibiotalar joint [6-10], decrease the contact pressure of the medial part of the tibiotalar joint $[11,12]$, and even reverse the radiological ankle osteoarthritis stages $[5,13,14]$.

However, the role of the talar tilt (TT) angle on SMOT is controversial. Some studies reported that increased 
preoperative TT angles were correlated with increased postoperative TT angles [5, 14-16], and large postoperative $\mathrm{TT}$ angles were positively correlated with treatment failure $[14,16]$. Although some authors reported that no radiological outcomes seemed to have a significant influence on the clinical outcomes [17], many doctors and patients are still worried about the potential for poor outcomes.

Ankle joint distraction arthroplasty was first reported in 1995 by van Valburg and his colleagues [18] and has evolved as an option for cartilage preservation in patients with osteoarthritis $[19,20]$. Although distraction arthroplasty cannot correct the bony deformities, it may shift the force from the hindfoot to the valgus at the joint level and give the medial structure a distraction. We found that in our distraction patients, the increased TT angles were corrected and maintained during follow-up [21]. Therefore, we evaluated the use of medial distraction arthroplasty (MDA) in our SMOT patients to correct their increased TT angles, and we observed positive outcomes [18]. Thus, we hypothesize that distraction arthroplasty may play a role in realignment surgery and may help restore the congruence of the ankle joint. The purpose of the current study was to retrospectively analyze and compare the clinical and radiological outcomes of SMOT with or without MDA for the treatment of varus ankle osteoarthritis with large preoperative TT angles.

\section{Methods}

The current study was approved by the research board of our hospital. The authors retrospectively studied the outcomes of SMOT with or without MDA in the treatment of varus ankle osteoarthritis with increased TT angles between January 2010 and October 2016. The inclusion criteria were as follows: (1) adults who were at least 18 years of age; (2) patients with a tibial articular surface (TAS) angle of less than $84^{\circ}$; (3) patients with varus ankle osteoarthritis; (4) patients with clinical symptoms, such as pain with walking and limitation of daily and recreational activities; (5) patients with a TT angle larger than $5^{\circ}$ [15]; (6) patients who were treated with SMOT with or without MDA; and (7) patients with at least 2 years of follow-up. The exclusion criteria were as follows: (1) patients with neurological disorders, (2) patients with rheumatoid arthritis, (3) patients with Charcot arthropathy, (4) patients with Charcot-Marie-Tooth deformity, (5) patients with acute or chronic infections of the ankle joint, and (6) patients who required reoperation after SMOT failure.

Finally, 16 cases in the SMOT group and 18 cases in the SMOT with MDA group were included in the study. There were 11 males and 23 females, and the mean age was 54.8 (range, 23-77) years. According to the modified Takakura ankle osteoarthritis stage, there were 13 patients with stage $3 \mathrm{a}$ osteoarthritis, 19 patients with stage $3 \mathrm{~b}$ osteoarthritis, and 2 patients with stage 4 . The basic information of the included patients is listed in Table 1. Between the 2 groups, there were more cases of autografts in the SMOT group and more allografts in the SMOT with MDA group $(P=0.014)$. The mean follow-up time in the SMOT group was longer than in the SMOT with MDA group $(P<0.001)$. There were no significant differences among the other information with the available numbers.

\section{Operative technique}

All of the included patients were treated with medial opening wedge SMOT. The surgical technique of SMOT for fibular osteotomy has been well described in a previous study [13]. The tibial osteotomy was approximately $5 \mathrm{~cm}$ proximal to the medial malleolar tip. Before osteotomy, a K-wire was placed from the medial malleolus to the lateral cortex to guide the osteotomy. The osteotomy plane slightly inclined from the medial-superior to the lateral-inferior and ended at the syndesmosis level. Subsequently, the osteotomy was performed with the use of

Table 1 Basic information and preoperative parameters of the two groups

\begin{tabular}{llll}
\hline & SMOT $(n=16)$ & $\begin{array}{l}\text { SMOT with MDA } \\
(n=18)\end{array}$ & $P$ value \\
\hline Male/female & $5 / 11$ & $6 / 12$ & 0.897 \\
Age, year & $53.4 \pm 10.2$ & $56.2 \pm 11.9$ & 0.470 \\
Left/right & $6 / 10$ & $5 / 13$ & 0.717 \\
Fibular osteotomy & 8 & 7 & 0.515 \\
Brostrom procedure & 3 & 5 & 0.693 \\
Calcaneal osteotomy & 1 & 2 & 0.998 \\
Takakura stage 3a/3b/4 & $7 / 8 / 1$ & $6 / 11 / 1$ & 0.805 \\
Auto-/allograft & $11 / 5$ & $4 / 14$ & 0.014 \\
Follow-up, month & $61.4 \pm 20.6$ & $35.6 \pm 15.3$ & $<0.001$ \\
Preoperative outcomes & & & 0.683 \\
AOFAS score, point & $47.3 \pm 14.9$ & $49.2 \pm 12.0$ & 0.311 \\
AOS pain, point & $5.6 \pm 0.9$ & $5.9 \pm 0.8$ & 0.389 \\
AOS function, point & $5.9 \pm 1.1$ & $6.2 \pm 0.9$ & 0.700 \\
ROM of ankle, degree & $34.7 \pm 7.8$ & $35.7 \pm 7.2$ & \\
Preoperative radiological & parameters (degree) & 0.7578 \\
TAS & $80.8 \pm 3.0$ & $81.6 \pm 2.2$ & 0.378 \\
TT & $11.2 \pm 3.4$ & $12.3 \pm 4.0$ & 0.397 \\
TMM & $32.6 \pm 7.3$ & $34.9 \pm 8.6$ & 0.410 \\
TC & $71.2 \pm 2.9$ & $69.5 \pm 4.1$ & 0.177 \\
TLS & $76.8 \pm 3.6$ & $75.4 \pm 3.4$ & 0.252 \\
HFA* & $16.7 \pm 4.4$ & $17.4 \pm 5.3$ & 0.755 \\
\hline
\end{tabular}

SMOT supramalleolar osteotomy, MDA medial distraction arthroplasty, AOFAS American Orthopaedic Foot and Ankle Society ankle-hindfoot score, AOS the Ankle Osteoarthritis Scale, ROM range of motion, TAS tibial articular surface angle, $T$ talar tilt angle, TMM tibial medial malleolar angle, $T C$ tibiocrural angle, TLS tibial lateral surface angle, HFA hindfoot alignment angle *The case number in SMOT group was 8, and in SMOT with MDA group was 14 
a wide saw blade, and the lateral cortex was carefully preserved. According to the preoperative plan, the aim for the TAS angle was 90 to $92^{\circ}$, and the aim for the tibial lateral surface (TLS) angle was 80 to $85^{\circ}$. The patient chose whether an iliac autograft or allograft was used to fill the tibial osteotomy site. The resulting sharp spike of the medial distal tibia bone from the opening wedge was removed, if necessary. Then, the osteotomy site was internally fixed with the use of a medial plate.

If the talocrural (TC) angle decreased by more than $5^{\circ}$ in comparison with the uninjured site [22], the fibula had a rotational deformity, or there was interference with the reduction of the tibial plafond and talus, then a fibular osteotomy was performed with a lateral approach at the same level or higher than that for the tibial osteotomy. The fibular osteotomy sites were internally fixed with plates.

If the patient had chronic ankle joint instability or was unstable after lateral osteophyte debridement, a modified Brostrom procedure was used to ensure the lateral stability of the ankle joint. If the patient still had varus deformity of the hindfoot after SMOT, a calcaneal osteotomy was used to further improve the lateral movement of the weight-bearing site in the hindfoot.

In the SMOT with MDA group, after the SMOT incisions were closed, an external fixator was applied. One pin was placed into the calcaneus under fluoroscopy, one half pin was placed into the talus from the medial side to the lateral side, and one or two additional pins were placed into the tibia. A fixed external fixator, which allowed further distraction without permitting ankle joint motion, was applied. With the distraction of the talus pin during the operation, the TT angle was corrected to $0^{\circ}$ or negative degrees and was verified fluoroscopically.
The postoperative rehabilitation protocol included active and passive motion exercises of the ankle and midfoot and forefoot joints, isotonic and isometric exercises of the leg, and the use of a night splint beginning on the second postoperative day in the SMOT group. Patients were permitted to be partially weight-bearing 6 weeks postoperation. Full weight-bearing activities began after the osteotomy site achieved bony union that was confirmed radiographically. The external fixator was used for 10 to 12 weeks in the SMOT with MDA group. The postoperative rehabilitation protocol in the SMOT with MDA group was similar to that of the SMOT group, except that weight-bearing actions and motions of the ankle joint were prohibited. After the external fixator was removed, the patient was permitted to begin partial weight-bearing activities for 1 month and then full weight-bearing activities after the osteotomy site achieved bony union, which was confirmed radiographically.

\section{Assessments}

The radiological evaluations visualized the TAS, TT, TC, and tibial medial malleolar (TMM) angles in an anteriorposterior ankle view, the TLS angle in a lateral ankle view, and the hindfoot alignment (HFA) angle in the Saltzman view (Fig. 1). All of the included measurements on the weight-bearing radiographs were performed by two observers independently.

The American Orthopaedic Foot and Ankle Society (AOFAS) ankle-hindfoot score and the Ankle Osteoarthritis Scale (AOS) were used to evaluate the functional outcomes preoperatively and postoperatively [23, 24]. Treatment failure was defined as the patient requiring reoperation because of the relative reasons for the initial operation. The
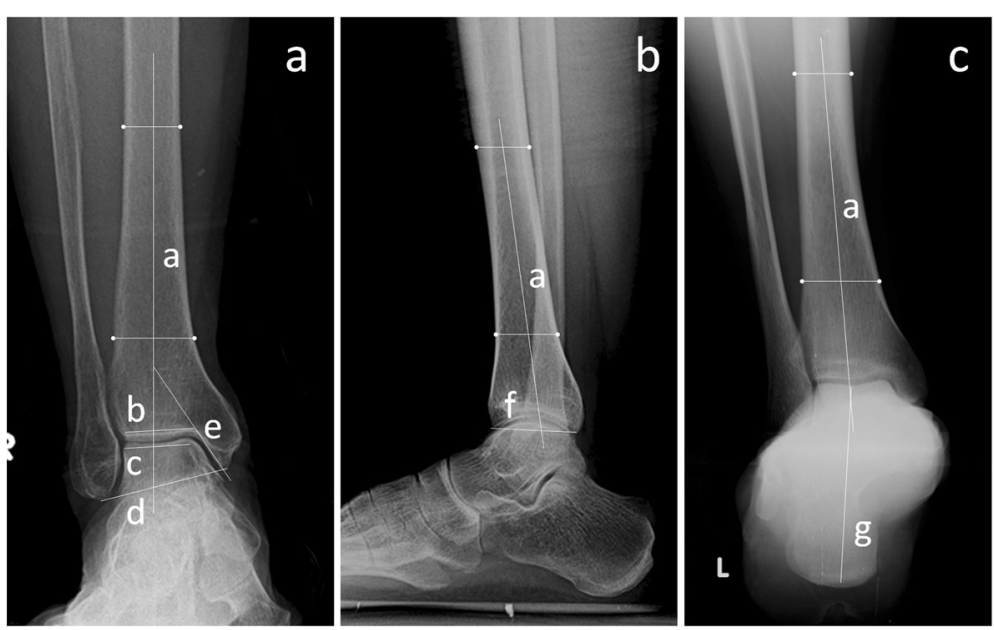

Fig. 1 Anterior-posterior view of the ankle (a). Tibial articular surface (TAS) angle, the angle between line a and b; talar tilt (TT) angle, the angle between line $b$ and $c$; talocrural (TC) angle, the angle between line $a$ and d; and tibial medial malleolar (TMM) angle, the angle between line $a$ and e. Lateral view of the ankle (b). The tibial lateral surface (TLS) angle, the angle between tibial axis line a and articular tangent line $\mathrm{f}$. Saltzman view of the ankle and the hindfoot alignment (HFA) angle, the line between a and $g$ (c) 
reoperations included osteotomy, arthrodesis, and arthroplasty; patients with no symptoms after hardware removal were not included. The functional outcomes and radiological parameters before failure relative to those after reoperation were included as the patients' final results.

To analyze the changes in the radiographical grade, stages 1, 2, 3a, 3b, and 4 of the modified Takakura classification system were assigned quantitative scores of 1,2 , 3,4 , and 5 , respectively.

\section{Statistical analyses}

Descriptive statistics were calculated as the means \pm standard deviation. Statistical analyses of the included data were performed using Student's $t$ test, Pearson's chi-square test, or Fisher's exact test with the level of significance set at $\alpha=0.05$. The statistical analyses were performed with SPSS 17.0 software (SPSS, Inc., Chicago, IL).

\section{Results}

\section{Functional and radiological improvement in the two} groups

All patients in both groups achieved bony unions without incision-related complications. No patient in either group had other soft tissue complications. Pin tract infections of the tibia occurred in two patients in the SMOT with MDA group. Both of these patients were treated with dressing changes and oral antibiotics; no patient needed early removal of the external fixator. When compared with the preoperative conditions, both groups achieved significant improvement in the AOFAS scores $(P<0.001$, Table 2$)$, as well as in the AOS pain and functional scores $(P<0.01)$. However, the range of motion (ROM) of the ankle joint was not significantly improved with the available numbers. The modified Takakura stages in both groups decreased significantly $(P<0.001)$. In both groups, all of the radiological parameters, including the TAS, TT, TMM, TC, TLS, and HFA angles, improved significantly $(P<0.05)$.

\section{Functional and radiological comparison between the two groups}

When comparing the postoperative functional outcomes of the two groups, the AOFAS scores and AOS pain and functional scores were not significantly different at the time of the final follow-up with the available numbers (Table 3). Three patients in the SMOT group underwent ankle arthrodesis at 17,26 , and 61 months postoperatively because of pain and dysfunction. No patients in the SMOT with MDA group required arthrodesis by the end of the follow-up time. However, the two groups demonstrated no significant differences in failure rate with the available numbers. Additionally, the postoperative modified Takakura stages were not significantly different with the available numbers.

When comparing the postoperative radiological parameters of the two groups, the TT angle in the SMOT with MDA group was significantly smaller than that of the SMOT group $(P=0.023)$. All of the TT angles in the SMOT with MDA group were corrected to normal angles $\left(\leq 4^{\circ}\right.$ [15], Fig. 2). However, the TT angles of five patients in the SMOT group were not corrected to normal angles $(P=0.016)$. Among these five patients,

Table 2 Comparison of the preoperative and last follow-up time functional outcomes and radiological parameters

\begin{tabular}{|c|c|c|c|c|c|c|}
\hline & \multicolumn{2}{|l|}{ SMOT $(n=16)$} & \multirow[t]{2}{*}{$P$ value } & \multicolumn{2}{|c|}{ SMOT with MDA $(n=18)$} & \multirow[t]{2}{*}{$P$ value } \\
\hline & Preoperation & Last follow-up & & Preoperation & Last follow-up & \\
\hline \multicolumn{7}{|l|}{ Functional outcomes } \\
\hline AOFAS, point & $47.3 \pm 14.9$ & $77.4 \pm 19.9$ & $<0.001$ & $49.2 \pm 12.0$ & $84.5 \pm 6.7$ & $<0.001$ \\
\hline AOS pain, point & $5.6 \pm 0.9$ & $3.5 \pm 2.3$ & 0.002 & $5.9 \pm 0.8$ & $2.4 \pm 0.6$ & $<0.001$ \\
\hline AOS function, point & $5.9 \pm 1.1$ & $3.6 \pm 2.0$ & $<0.001$ & $6.2 \pm 0.9$ & $2.9 \pm 0.9$ & $<0.001$ \\
\hline ROM of ankle, degree & $34.7 \pm 7.8$ & $36.4 \pm 7.6$ & 0.537 & $35.7 \pm 7.2$ & $37.8 \pm 6.3$ & 0.358 \\
\hline Takakura stage & $3.6 \pm 0.6$ & $1.9 \pm 1.0$ & $<0.001$ & $3.8 \pm 0.6$ & $1.4 \pm 0.6$ & $<0.001$ \\
\hline \multicolumn{7}{|l|}{ Radiological parameters } \\
\hline TAS & $80.8 \pm 3.0$ & $89.1 \pm 2.0$ & $<0.001$ & $81.6 \pm 2.2$ & $89.8 \pm 1.6$ & $<0.001$ \\
\hline$\pi$ & $11.2 \pm 3.4$ & $2.9 \pm 1.7$ & $<0.001$ & $12.3 \pm 4.0$ & $1.8 \pm 0.9$ & $<0.001$ \\
\hline TMM & $32.6 \pm 7.3$ & $27.7 \pm 4.6$ & 0.031 & $34.9 \pm 8.6$ & $28.5 \pm 6.2$ & 0.015 \\
\hline TC & $71.2 \pm 2.9$ & $77.9 \pm 2.8$ & $<0.001$ & $69.5 \pm 4.1$ & $76.9 \pm 3.7$ & $<0.001$ \\
\hline TLS & $76.8 \pm 3.6$ & $80.5 \pm 2.3$ & 0.002 & $75.4 \pm 3.4$ & $79.5 \pm 3.0$ & 0.001 \\
\hline $\mathrm{HFA}^{*}$ & $16.7 \pm 4.4$ & $4.2 \pm 2.6$ & $<0.001$ & $17.4 \pm 5.3$ & $3.1 \pm 1.8$ & $<0.001$ \\
\hline
\end{tabular}

SMOT supramalleolar osteotomy, MDA medial distraction arthroplasty, AOFAS American Orthopaedic Foot and Ankle Society ankle-hindfoot score, AOS the Ankle Osteoarthritis Scale, ROM range of motion, TAS tibial articular surface angle, $T T$ talar tilt angle, $T M M$ tibial medial malleolar angle, $T C$ tibiocrural angle, $T L S$ tibial lateral surface angle, HFA hindfoot alignment angle

*The case number in SMOT group was 8, and in SMOT with MDA group was 14 
Table 3 Functional outcomes and radiological parameters between the two groups at the last follow-up time

\begin{tabular}{llll}
\hline & SMOT $(n=16)$ & $\begin{array}{l}\text { SMOT with MDA } \\
(n=18)\end{array}$ & $P$ value \\
\hline Functional outcomes & & & \\
AOFAS, point & $77.4 \pm 19.9$ & $84.5 \pm 6.7$ & 0.163 \\
AOS pain, point & $3.5 \pm 2.3$ & $2.4 \pm 0.6$ & 0.059 \\
AOS function, point & $3.6 \pm 2.0$ & $2.9 \pm 0.9$ & 0.189 \\
ROM of ankle, degree & $36.4 \pm 7.6$ & $37.8 \pm 6.3$ & 0.561 \\
Failure rate & $18.8 \%(3 / 16)$ & 0 & 0.094 \\
Takakura stage & $1.9 \pm 1.0$ & $1.4 \pm 0.6$ & 0.083 \\
Radiological parameters & & & \\
TAS & $89.1 \pm 2.0$ & $89.8 \pm 1.6$ & 0.266 \\
TT & $2.9 \pm 1.7$ & $1.8 \pm 0.9$ & 0.023 \\
TMM & $27.7 \pm 4.6$ & $28.5 \pm 6.2$ & 0.675 \\
TC & $77.9 \pm 2.8$ & $76.9 \pm 3.7$ & 0.386 \\
TLS & $80.5 \pm 2.3$ & $79.5 \pm 3.0$ & 0.288 \\
HFA* & $4.2 \pm 2.6$ & $3.1 \pm 1.8$ & 0.254 \\
TT not corrected & $31.3 \%(5 / 16)$ & 0 & 0.016 \\
\hline
\end{tabular}

SMOT supramalleolar osteotomy, MDA medial distraction arthroplasty, AOFAS American Orthopaedic Foot and Ankle Society ankle-hindfoot score, AOS the Ankle Osteoarthritis Scale, ROM range of motion, TAS tibial articular surface angle, $\Pi$ talar tilt angle, $T M M$ tibial medial malleolar angle, $T C$ tibiocrural angle, $T L S$ tibial lateral surface angle, HFA hindfoot alignment angle *The case number in SMOT group was 8, and in SMOT with MDA group was 14

two patients had fused joints, two were still symptomatic and treated conservatively, and only one reported a good outcome. The other postoperative radiological parameters, including TAS, TMM, TC, TLS, and HFA angles, were not significantly different with the numbers available.

\section{Discussion}

Osteoarthritis is a slowly progressive degenerative joint disorder that, in most cases, is diagnosed at a late stage after the onset of accompanying clinical symptoms. Ankle joint osteoarthritis is one of the most common joint diseases and is a significant source of pain and disability for middle-aged and elderly people throughout the world [25]. Joint-sacrificing procedures, including total ankle replacement and arthrodesis, are used for painful end-stage ankle osteoarthritis. However, both procedures have disadvantages and are associated with limited long-term benefits [1,26, 27]. Realignment osteotomy, which is based on the theory that uneven pressure on the articular surface of the lower extremities may induce arthritis [3], is used to redistribute the weight-bearing pressure on the joint to delay the progression and development of osteoarthritis. The midterm results of SMOT showed good outcomes for pain relief, functional improvement, and a return to sports and recreational activities [5-10, 14, 16, 17, 22, 28-30]. However, the treatment is still controversial, especially in patients with increased preoperative TT angles.

Talar tilt is common in varus ankle osteoarthritis and is not a bony deformity that results in the incongruence of the tibiotalar joint. The initial reason for the increased TT angle may be due to a lateral collateral ligament injury of the ankle joint. However, this will result in a medial shift of the center of the talus and joint loading axis [12] and will increase the tension and expansion of the lateral soft tissue to further increase the deformity. Some authors reported that SMOT could significantly decrease the TT angle $[7,9,17,22,31]$; however, some did not observe the same decrease [6,14-16]. Tanaka et al. [14] reported that all patients with a preoperative TT
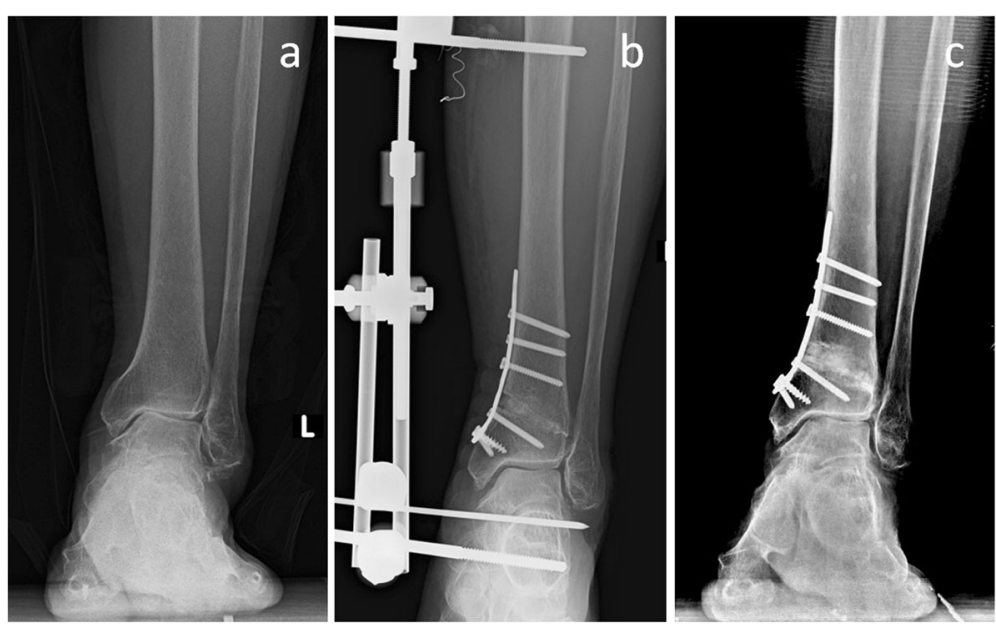

Fig. 2 A 62-year-old female patient. The preoperative anterior-posterior view showed stage $3 b$ varus ankle osteoarthritis (a). The patient was treated with supramalleolar osteotomy and medial distraction arthroplasty (b). The 1-year postoperative scan showed normal alignment of the ankle joint; the talar tilt angle was decreased to $1.7^{\circ}$, and the modified Takakura stage was improved to stage 1 (c) 
angle larger than $10^{\circ}$ in the joint space did not improve to a normal angle. Lee et al. [16] reported that the preoperative TT angle was correlated with the postoperative TT angle and recommended that the optimal threshold for predicting a large postoperative TT angle was a preoperative TT angle of $7.3^{\circ}$. Ahn et al. [6] reported that the TT angle was not significantly corrected after SMOT. In our SMOT cases, the TT angles of five $(31.3 \%)$ cases did not correct to a normal angle postoperatively, and two of the five (40\%) patients experienced treatment failure. Therefore, a challenge for surgeons is effectively correcting the increased TT angle to a normal angle in varus ankle osteoarthritis patients.

Joint distraction arthroplasty in the treatment of severe ankle osteoarthritis shows significant and prolonged improvement in pain and functional ability in open prospective studies as well as in a randomized controlled trial $[32,33]$. Our previous study reported that distraction arthroplasty using talus medial half pins could shift the force from the hindfoot to the valgus, thereby distracting the medial structure and relaxing the lateral structure [21]. It was expected that 3 months following distraction, the medial and lateral soft tissues would recover to some degree, and the TT correction would be maintained in some cases [1]. Based on this line of thinking, a fixed medial ankle joint distraction arthroplasty was used to further open the medial joint space and correct the TT angle in our later patients [34]. Tellisi et al. [35] reported 23 ankle joint distraction arthroplasty cases, and 6 of them were combined with SMOT to correct distal tibial deformities; however, no cases in this series focused on the increased TT angle. To date, no clinical study has reported the outcomes of SMOT combined with distraction arthroplasty for varus ankle osteoarthritis with increased TT angles.

To the best of our knowledge, this is the first study to report the functional and radiological outcomes of combining SMOT and distraction arthroplasty to correct varus ankle osteoarthritis with large varus TT angles and is the first study to compare the results of this technique with SMOT. According to the current results, SMOT alone has a high rate of failed correction of the TT angle (31.3\%, Fig. 3). However, in the SMOT with MDA group, the increased TT angles in all of the patients were corrected to normal angles, and the correction was maintained (Fig. 4). The mean postoperative TT angle in the SMOT with MDA group was significantly smaller than in the SMOT group.

The limitations of the current study include its retrospective design and the lack of information on the intraarticular changes. Additionally, we used more autografts in the SMOT group and more allografts in the SMOT with MDA group. This difference is because we used more allografts in our later patents to decrease the relative donor site complications of the autografts, and we did not find delayed unions or nonunions with the use of allografts. The follow-up time in the SMOT with MDA group was significantly shorter than that of the SMOT group, which may be

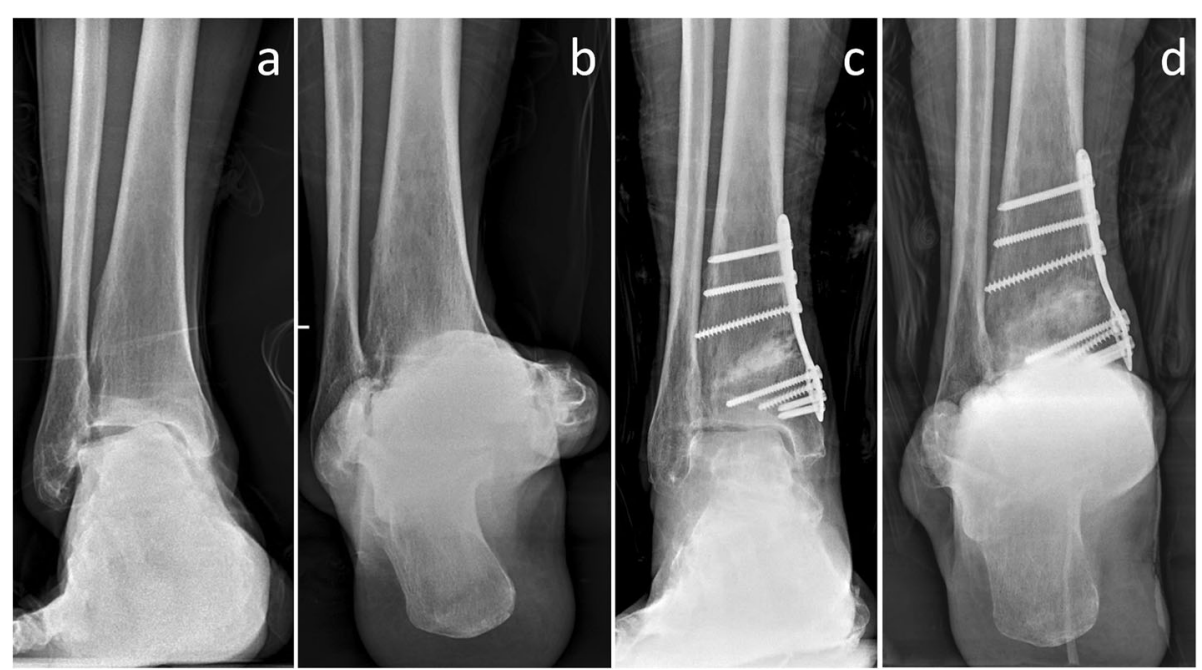

Fig. 3 A 58-year-old male patient. The preoperative anterior-posterior view (a) and Saltzman view (b) showed stage 3a varus ankle osteoarthritis. The preoperative talar tilt angle was $14.3^{\circ}$. The patient was treated with supramalleolar osteotomy and modified Brostrom procedures. The 1-year postoperative scans showed that the alignment was better than that of the preoperative conditions, the functional outcomes were improved, and the modified Takakura stage was improved to stage 2 (c) $\mathbf{d}$ ), but the talar tilt angle was still larger than normal $\left(6.4^{\circ}\right)$ 


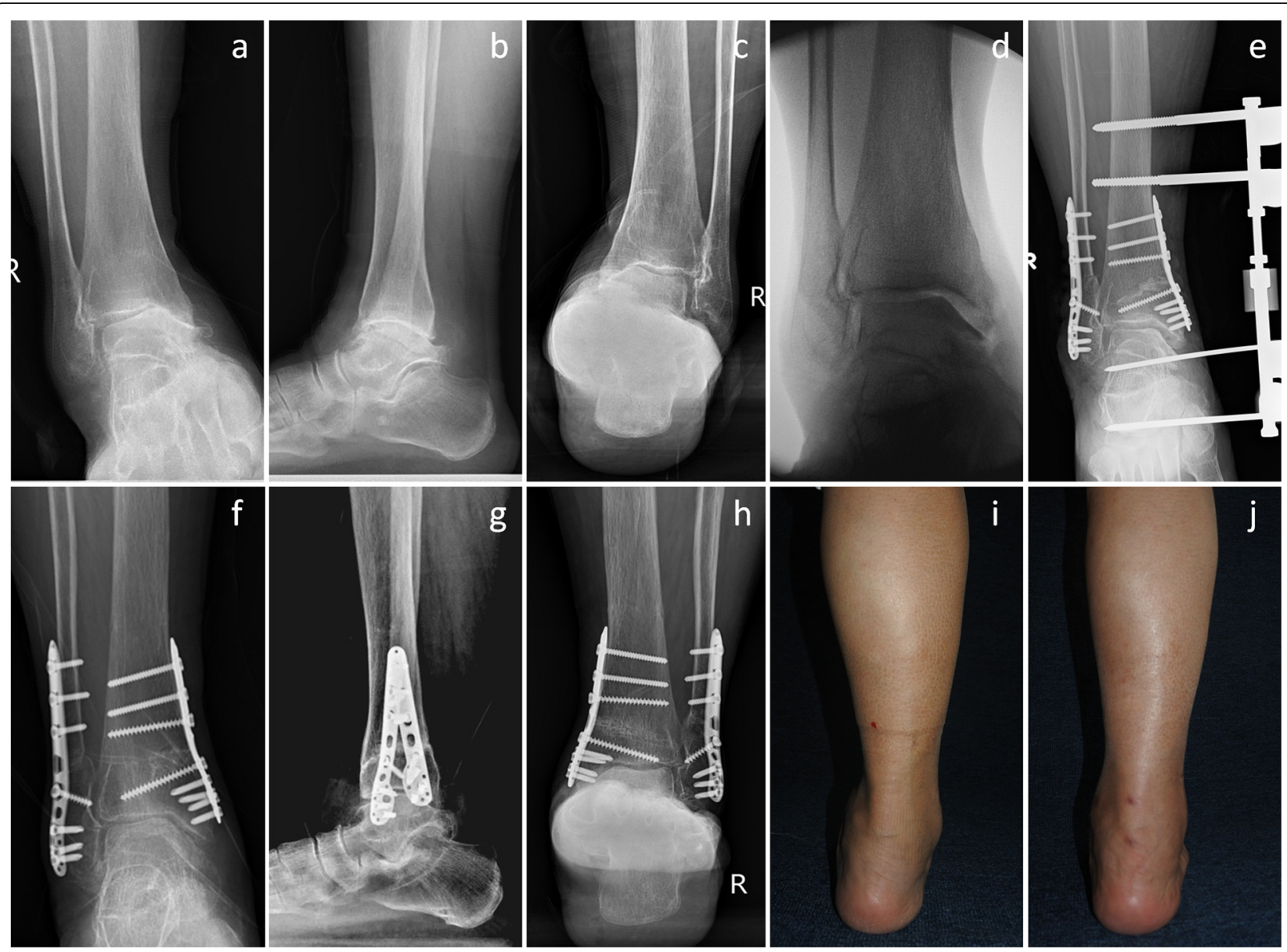

Fig. 4 A 64-year-old female patient. The preoperative anterior-posterior view (a), lateral view (b), and Saltzman view (c) showed stage 3a varus ankle osteoarthritis. The preoperative talar tilt angle was $13.2^{\circ}$. The valgus stress view showed that the medial malleolus joint space could be opened, and the talar tilt could be corrected (d). The patient was treated with supramalleolar osteotomy, fibular osteotomy, and medial distraction arthroplasty (e). The 1-year postoperative scans showed that the normal alignment was maintained; the talar tilt angle was decreased to $1.4^{\circ}$, and the modified Takakura stage was improved to stage $1(\mathbf{f}, \mathbf{g}, \mathbf{h})$. The preoperative hindfoot showed severe varus deformity (i), and the alignment was corrected to normal angles postoperatively (j)

because we just started to use this technique (SMOT with MDA) 5 years previously. Other limitations included a relatively small sample size and a large age range in the included patients, which may result in heterogeneity. Although the outcomes will change over time, our early results confirmed that the functional outcome of the SMOT procedure is good in terms of alleviating pain relief, correcting the malalignment, and reducing the signs of varus ankle osteoarthritis patients with increased TT angles. In addition, the combined use of SMOT and distraction arthroplasty could better correct the TT angle.

\section{Conclusions}

In conclusion, SMOT combined with MDA can improve the clinical and radiological outcomes of varus ankle osteoarthritis with large TT angles. This procedure may be helpful to restore the weight-bearing alignment of the ankle joint and to correct the TT angle that opens the medial ankle joint space. However, well-designed prospective comparative studies are still needed to further confirm the outcomes of medial distraction for increased TT angles.

\section{Abbreviations}

AOFAS: American Orthopaedic Foot and Ankle Society; AOS: Ankle Osteoarthritis Scale; HFA: Hindfoot alignment angle; MDA: Medial distraction arthroplasty; ROM: Range of motion; SMOT: Supramalleolar osteotomy; TAS: Tibial articular surface angle; TC: Tibiocrural angle; TLS: Tibial lateral surface angle; TMM: Tibial medial malleolar angle; TT: Talar tilt angle

\section{Acknowledgements}

Not applicable.

\section{Funding}

The author(s) disclosed receipt of the following financial support for the research, authorship, and/or publication of this article: China postdoctoral 
science foundation funding project (2017 M613178), Shaanxi Province Natural Science Foundation funding project (2018JM7112), Shaanxi Province Postdoctoral Science Foundation funding project (2017BSHQYXMZZ13).

\section{Availability of data and materials}

The data of this study were real and were performed in the SPSS 17.0 software (SPSS Inc., Chicago, IL). The statistical results of the data are presented in this main paper. The images of the case examples are depicted in this research article. All of the data are available in contact with the corresponding author.

\section{Authors' contributions}

ZHM and LXJ designed the study, analyzed the data, and wrote the manuscript. WXD and ZY participated in the design of the study and analyzed the data. $L J Q, L P L, L Y$, and $L J$ collected the data, followed up the patients, and helped in writing the manuscript. All authors read and approved the final manuscript.

\section{Ethics approval and consent to participate}

This study has been approved by the ethical committee of Honghui Hospital. We have obtained the consent to participate from the participants.

\section{Consent for publication}

Not applicable.

\section{Competing interests}

The authors declared no potential conflicts of interest with respect to the research, authorship, and/or publication of this article.

\section{Publisher's Note}

Springer Nature remains neutral with regard to jurisdictional claims in published maps and institutional affiliations.

Received: 11 January 2019 Accepted: 26 April 2019

Published online: 06 May 2019

\section{References}

1. Fuchs S, Sandmann C, Skwara A, Chylarechi C. Quality of life 20 years after arthrodesis of the ankle. A study of adjacent joints. J Bone Joint Surg Br. 2003:85:994-8.

2. Horisberger M, Hintermann B, Valderrabano V. Alterations of plantar pressure distribution in posttraumatic end-stage ankle osteoarthritis. Clin Biomech (Bristol, Avon). 2009:24:303-7.

3. Salter RB, Field P. The effects of continuous compression on living articular cartilage. J Bone Joint Surg Am. 1960;42:31-49.

4. Speed JS, Boyd HB. Operative reconstruction of malunited fractures about the ankle joint. J Bone Joint Surg Am. 1936;18:270-86.

5. Takakura Y, Tanaka Y, Kumai T, Tamai S. Low tibial osteotomy for osteoarthritis of the ankle. Results of a new operation in 18 patients. J Bone Joint Surg Br. 1995;77:50-4.

6. Ahn TK, Yi Y, Cho JH, Lee WC. A cohort study of patients undergoing distal tibial osteotomy without fibular osteotomy for medial ankle arthritis with mortise widening. J Bone Joint Surg Am. 2015;97:381-8.

7. Colin F, Bolliger L, Horn Lang T, Knupp M, Hintermann B. Effect of supramalleolar osteotomy and total ankle replacement on talar position in the varus osteoarthritic ankle: a comparative study. Foot Ankle Int. 2014;35: 445-52.

8. Haraguchi N, Ota K, Tsunoda N, Seike K, Kanetake Y, Tsutaya A. Weight bearing line analysis in supramalleolar osteotomy for varus-type osteoarthritis of the ankle. J Bone Joint Surg Am. 2015;97:333-9.

9. Nüesch C, Huber C, Paul J, Henninger HB, Pagenstert G, Valderrabano V, et al. Mid- to long-term clinical outcome and gait biomechanics after realignment surgery in asymmetric ankle osteoarthritis. Foot Ankle Int. 2015; 36:908-18.

10. Pagenstert G, Leumarm A, Hintermann B, Valderrabano V. Sports and recreation activity of varus and valgus ankle osteoarthritis before and after realignment surgery. Foot Ankle Int. 2008;29:985-93.

11. Stufkens SA, van Bergen CJ, Blankevoort L, van Dijk CN, Hintermann B, Knupp M. The role of the fibula in varus and valgus deformity of the tibia: a biomechanical study. J Bone Joint Surg Br. 2011;93:1232-9.
12. Zhao HM, Liang XJ, Li Y, Yu GR, Niu WX, Zhang Y. The role of fibular for supramalleolar osteotomy in treatment of varus ankle arthritis: a biomechanical and clinical study. J Orthop Surg Res. 2016;11:127.

13. Hongmou Z, Xlaojun L, Yi L, Hongliang L, Wang J, Cheng L. Supramalleolar osteotomy with or without fibular osteotomy for varus ankle arthritis. Foot Ankle Int. 2016;37:1001-7.

14. Tanaka Y, Takakura Y, Hayashi K, Taniguchi A, Kumai T, Sugimoto K. Low tibial osteotomy for varus-type osteoarthritis of the ankle. J Bone Joint Surg Br. 2006:88:909-13.

15. Knupp M, Stufkens SA, Bolliger L, Barg A, Hintermann B. Classification and treatment of supramalleolar deformities. Foot Ankle Int. 2011:32:1023-31.

16. Lee WC, Moon JS, Lee K, Byun WJ, Lee SH. Indications for supramalleolar osteotomy in patients with ankle osteoarthritis and varus deformity. J Bone Joint Surg Am. 2011;93:1243-8.

17. Kim YS, Park EH, Koh YG, Lee JW. Supramalleolar osteotomy with bone marrow stimulation for varus ankle osteoarthritis: clinical results and secondlook arthroscopic evaluation. Am J Sports Med. 2014;42:1558-66.

18. Van Valburg AA, van Roermund PM, Lammens J. Can Ilizarov joint distraction delay the need for an arthrodesis of the ankle? A preliminary report. J Bone Joint Surg Br. 1995;77:720-5.

19. Jessica CR, Beachler JA. Distraction arthroplasty compared to other cartilage preservation procedures in patients with post-traumatic arthritis: a systematic review. Strategies Trauma Limb Reconstr. 2018;13:61-7.

20. Paley D, Lamm BM, Purohit RM, Specht SC. Distraction arthroplasty of the ankle--how far can you stretch the indications? Foot Ankle Clin N Am. 2008; 13:471-84.

21. Zhao HM, Qu WQ, Li Y, Liang XJ, Ning N, Zhang Y, et al. Functional analysis of distraction arthroplasty in the treatment of ankle osteoarthritis. J Orthop Surg Res. 2017;12:18

22. Pagenstert G, Hintermann B, Barg A, Leumann A, Valderrabano V. Realignment surgery as alternative treatment of varus and valgus ankle osteoarthritis. Clin Orthop Relat Res. 2007;462:156-68.

23. Domsic RT, Saltzman CL. Ankle osteoarthritis scale. Foot Ankle Int. 1998:19: 466-71

24. Kitaoka HB, Alexander IJ, Adelaar RS, Nunley JA, Myerson MS, Sanders M. Clinical rating systems for the ankle-hindfoot, midfoot, hallux, and lesser toes. Foot Ankle Int. 1994:15:349-53.

25. Daniels T, Thomas R. Etiology and biomechanics of ankle arthritis. Foot Ankle Clin N Am. 2008:13:341-52.

26. Gross CE, Lewis JS, Adams SB, Easley M, DeOrio JK, Nunley JA 2nd. Secondary arthrodesis after total ankle arthroplasty. Foot Ankle Int. 2016;37:709-14.

27. Zhao HM, Yang YF, Yu GR, Zhou JQ. A systematic review of outcome and failure rate of uncemented Scandinavian total ankle replacement. Int Orthop. 2011:35:1751-8.

28. Harstall R, Lehmann O, Krause F, Weber M. Supramalleolar lateral closing wedge osteotomy for the treatment of varus ankle arthrosis. Foot Ankle Int. 2007;28:542-8.

29. Mann HA, Filippi J, Myerson MS. Intra-articular opening medial tibial wedge osteotomy (plafond-plasty) for the treatment of intra-articular varus ankle arthritis and instability. Foot Ankle Int. 2012:33:255-61.

30. Stamatis ED, Cooper PS, Myerson MS. Supramalleolar osteotomy for the treatment of distal tibial angular deformities and arthritis of the ankle joint. Foot Ankle Int. 2003;24:754-64.

31. Kobayashi H, Kageyama Y, Shido Y. Treatment of varus ankle osteoarthritis and instability with a novel mortise-plasty osteotomy procedure. J Foot Ankle Surg. 2016;55:60-7.

32. Nguyen MP, Pedersen DR, Gao Y, Saltzman CL, Amendola A. Intermediateterm follow-up after ankle distraction for treatment of end-stage osteoarthritis. J Bone Joint Surg Am. 2015:97:590-6.

33. Saltzman CL, Hillis SL, Stolley MP, Anderson DD, Amendola A. Motion versus fixed distraction of the joint in the treatment of ankle osteoarthritis: a prospective randomized controlled trial. J Bone Joint Surg Am. 2012;94:961-70.

34. Zhao HM, Liang XJ, Li Y, Ning N, Lu J. Supramalleolar osteotomy with distraction arthroplasty in treatment of varus ankle osteoarthritis with large talar tilt angle: a case report and literature review. J Foot Ankle Surg. 2017; 56:1125-8.

35. Tellisi N, Fragomen AT, Rozbruch SR, O'Malley MJ, Rozbruch SR. Joint preservation of the osteoarthritic ankle using distraction arthroplasty. Foot Ankle Int. 2009;30:318-25. 